\title{
Scalable and Tunable Periodic Graphene Nanohole Arrays for Mid-Infrared Plasmonics
}

Gopalan, Kavitha K.; Paulillo, Bruno; Mackenzie, David M. A.; Rodrigo, Daniel; Bareza, Nestor; Whelan, Patrick R.; Shivayogimath, Abhay; Pruneri, Valerio

\section{Published in:}

Nano Letters

Link to article, DOI:

10.1021/acs.nanolett.8b02613

Publication date:

2018

Document Version

Publisher's PDF, also known as Version of record

Link back to DTU Orbit

Citation (APA):

Gopalan, K. K., Paulillo, B., Mackenzie, D. M. A., Rodrigo, D., Bareza, N., Whelan, P. R., Shivayogimath, A., \& Pruneri, V. (2018). Scalable and Tunable Periodic Graphene Nanohole Arrays for Mid-Infrared Plasmonics.

Nano Letters, 18(9), 5913-5918. https://doi.org/10.1021/acs.nanolett.8b02613

\section{General rights}

Copyright and moral rights for the publications made accessible in the public portal are retained by the authors and/or other copyright owners and it is a condition of accessing publications that users recognise and abide by the legal requirements associated with these rights.

- Users may download and print one copy of any publication from the public portal for the purpose of private study or research.

- You may not further distribute the material or use it for any profit-making activity or commercial gain

- You may freely distribute the URL identifying the publication in the public portal 


\title{
Scalable and Tunable Periodic Graphene Nanohole Arrays for Mid- Infrared Plasmonics
}

\author{
Kavitha K. Gopalan, ${ }^{\dagger}$ Bruno Paulillo, ${ }^{\dagger}$ David M.A. Mackenzie, ${ }^{\perp, \ddagger}$ Daniel Rodrigo, ${ }^{\dagger}$ Nestor Bareza, ${ }^{\dagger}$ \\ Patrick R. Whelan, ${ }^{\perp}$ (๑) Abhay Shivayogimath, ${ }^{\perp}$ and Valerio Pruneri*, ${ }^{*}, \#$ \\ ${ }^{\dagger}$ ICFO-Institut de Ciencies Fotoniques, The Barcelona Institute of Science and Technology, 08860 Castelldefels, Spain \\ ${ }^{\perp}$ Center for Nanostructured Graphene (CNG), Technical University of Denmark, DK-2800 Kgs. Lyngby, Denmark \\ ${ }^{\ddagger}$ Department of Micro- and Nanotechnology (DTU Nanotech), Technical University of Denmark, DK-2800 Kgs. Lyngby, Denmark \\ \#ICREA-Institució Catalana de Recerca i Estudis, Avançats Passeig Lluís Companys, 23, 08010 Barcelona, Spain
}

\section{Supporting Information}

\begin{abstract}
Despite its great potential for a wide variety of devices, especially mid-infrared biosensors and photodetectors, graphene plasmonics is still confined to academic research. A major reason is the fact that, so far, expensive and lowthroughput lithography techniques are needed to fabricate graphene nanostructures. Here, we report for the first time a detailed experimental study on electrostatically tunable graphene nanohole array surfaces with periods down to $100 \mathrm{~nm}$, showing clear plasmonic response in the range $\sim 1300-1600 \mathrm{~cm}^{-1}$, which
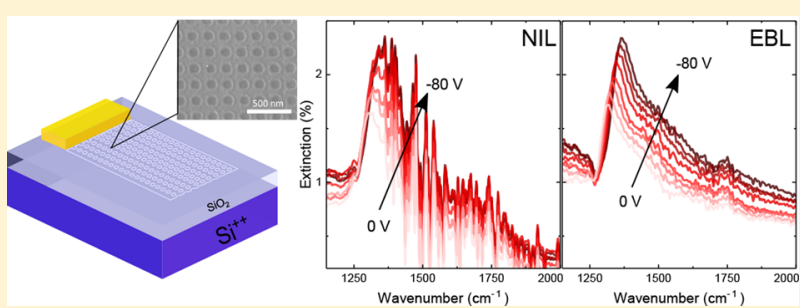
can be fabricated by a scalable nanoimprint technique. Such large area plasmonic nanostructures are suitable for industrial applications, for example, surface-enhanced infrared absorption (SEIRA) sensing, as they combine easy design, extreme field confinement, and the possibility to excite multiple plasmon modes enabling multiband sensing, a feature not readily available in nanoribbons or other localized resonant structures.
\end{abstract}

KEYWORDS: Graphene plasmonics, plasmonic crystals, SEIRA, large-scale nanopatterning, nanoimprint lithography

$\mathrm{T}$ hanks to its unique extreme subwavelength confinement and tunability, graphene plasmonics is relevant for many applications, including infrared detection, enhanced infrared absorption, metamaterials and chemical sensing. ${ }^{1-5}$ In particular, graphene plasmonics has shown great potential in the mid-infrared (mid-IR) wavelength region, from a few to several tens of micrometers, where it allows, for example, highly sensitive and specific detection of molecular species via their vibrational fingerprints and photodetection of thermal radiation. ${ }^{6,7}$

So far, graphene plasmonics has been mainly implemented in nanopatterned graphene ribbons, disks, or rings, producing localized surface plasmon resonances (LSPRs). In particular, graphene nanoribbon arrays have proven to be a robust platform for sensing of biomolecules in the mid-IR with high sensitivity and over an unprecedentedly wide tunable spectral range. ${ }^{6}$ In such systems, the spectral position of the plasmonic resonance is mainly set by the lateral dimension of the graphene nanoribbons while their spatial arrangement to form a large metasurface allows one to obtain an exploitable plasmonic response in the far-field by adding up the contributions from thousands of LSPR uncoupled resonators.

As an alternative, band engineering of graphene plasmons in a periodically patterned continuous graphene sheet has been proposed at far-infrared frequencies in so-called graphene plasmonic crystals or antidot or nanohole arrays. ${ }^{8-10}$
A periodic array of graphene nanoholes offers the unique feature of being an electrically connected surface, still maintaining the enhanced radiation absorption due to the excitation of graphene plasmons. It behaves as both a transparent conductive electrode and a plasmonic absorber. Moreover, due to its simple geometry, it is suitable for largescale fabrication nanopatterning relying, for instance, on nanoimprint or colloidal lithography. ${ }^{11-13}$ On a more fundamental level, this platform has recently been proposed to investigate topologically protected plasmon modes in the infrared. ${ }^{14}$

In this work, for the first time to our knowledge, we report a detailed experimental demonstration of large area, gatetunable, graphene nanohole arrays (GNHAs) operating at mid-IR wavelengths. We demonstrate experimentally that such nanostructured graphene surfaces behave as plasmonic crystals and support multiband resonances. In such nanostructures, one can tune the response by changing the geometrical parameters and applying an electrical voltage. Remarkably, we also show that functional, gate-tunable GNHAs can be successfully fabricated with large-scale nanoimprint lithography

Received: June 27, 2018

Revised: August 13, 2018

Published: August 16, 2018 
(a)

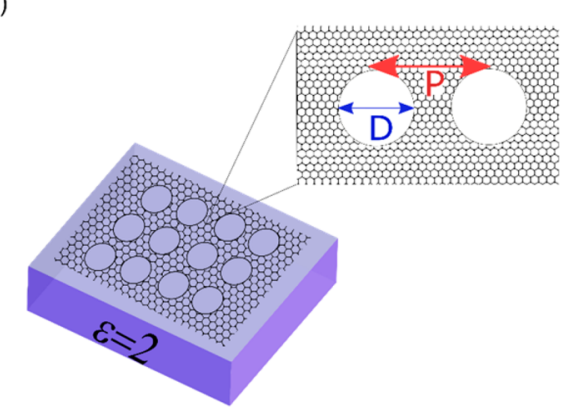

(c)

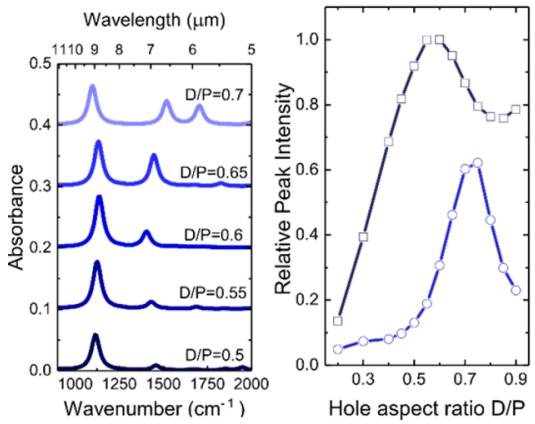

(b) Wavelength $(\mu \mathrm{m})$

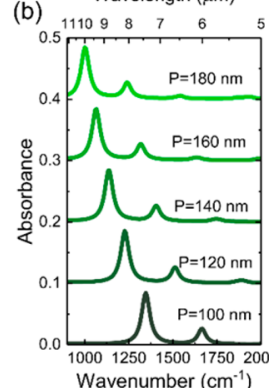

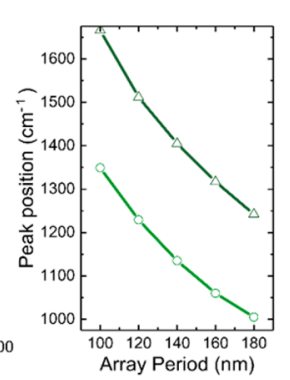

(d)

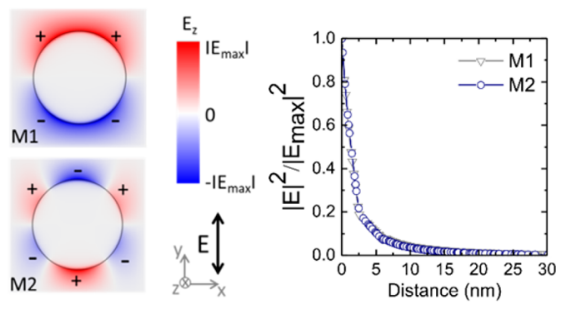

Figure 1. Simulations and theory of GNHAs. (a) Schematic of a GNHA highlighting the geometric parameters, $P$ and $D$. (b) (left) Absorption spectra (stacked) as a function of the array period $P$ for a fixed aspect ratio $D / P=0.6\left(E_{\mathrm{F}}=0.3, \tau=100 \mathrm{fs}\right)$ and (right) position of the plasmonic peak as a function of array period $P$. (c) (left) Absorption spectra (stacked) as a function of the hole aspect ratio $D / P$ for a fixed period $P=140 \mathrm{~nm}$ $\left(E_{\mathrm{F}}=0.3, \tau=100 \mathrm{fs}\right)$ and (right) relative peak intensity for the two visible plasmonic modes. The two modes become strongly coupled at $D / P \approx$ 0.7. (d) Out of plane $\left(E_{z}\right)$ field profile and field intensity decay $|E|^{2} /\left|E_{\max }\right|^{2}$ for $M 1$ and $M 2$ modes. In all these simulations, the substrate has $\varepsilon=2$.

(NIL) over wafer-scale areas, paving the way to low-cost midIR plasmonic sensors.

A nanostructured surface made of graphene nanoholes of diameter $D$ arranged in a square lattice of period $P$ is sketched in Figure 1a. The photonic band structure of such a plasmonic crystal was theoretically studied in ref 15 . It was found that, due to the extreme subwavelength confinement of the graphene plasmons, only the modes lying near the gamma point and whose symmetry matches the one of the impinging light field can be excited from the far field. We performed fullwave electromagnetic simulations using a commercial finite element package (Ansys HFSS) to study the response of the system under far-field excitation at mid-IR wavelengths as a function of the geometrical parameters (simulation details in Methods). In all the simulations in Figure 1, the values of the graphene Fermi level and relaxation time are $E_{\mathrm{F}}=0.3 \mathrm{eV}$ and $\tau$ $=100 \mathrm{fs}$, respectively, and the substrate has $\varepsilon=2$. The aim of such study is to derive useful rules of thumb to design the plasmonic response for applications in the mid-IR, such as chemical sensing. Figure $1 b, c$ shows the role of the two geometric parameters $P$ and $D$ in tailoring the optical modes of the system. In the left panel of Figure $1 \mathrm{~b}$, the simulated mid-IR absorption spectra under normal incidence for different $P$ and fixed aspect ratio $D / P=0.6$ are shown. In the spectra, we observe two dominant modes whose resonant frequencies as a function of the array period are plotted in the right panel of Figure $1 \mathrm{~b}$. These are identified as the $(1,0)$ and $(1,1)$ diffraction orders of the nanohole square lattice. ${ }^{10}$ The spectral position of the fundamental plasmon mode corresponds to the condition where the graphene plasmon wavevector matches the first diffraction order of the array, that is, $\lambda_{\mathrm{p}} \approx P$ where the plasmon effective wavelength is almost 2 orders of magnitude smaller than the free-space wavelength (typically $\lambda_{\mathrm{p}} \approx \lambda_{0} / 70$ in the mid-IR range). ${ }^{3}$ If we want to set the plasmon edge around $\lambda_{0}=7 \mu \mathrm{m}$ (e.g., for chemical sensing of a specific vibrational band), then $P \approx \lambda_{\mathrm{p}} \approx 100 \mathrm{~nm}$. The effective confinement of the two modes can be inferred from the slope of the $\lambda-P$ dispersion, yielding $\lambda_{0} / 70$ for the fundamental mode (M1) and $\lambda_{0} / 60$ for the higher order mode (M2). In Figure 1c, we report the simulated absorption spectra under normal incidence for different hole aspect ratio $D / P$ and fixed period $P$. For the considered $D / P$ values, we can see that the fundamental mode does not change substantially in position or intensity, whereas the visibility of M2 is greatly affected by $D$. The role of this parameter is better illustrated in the right panel of Figure 1c where the relative peak intensity for M1 and M2 (normalized to the maximum intensity of M1) is traced as a function of $D /$ $P$. We focus on $D / P$ values ranging from 0.3 to 0.7 , which corresponds to dimensions compatible with the available nanopatterning methods. We can see that for increasing hole size the fundamental M1 mode grows in intensity reaching its maximum around $D / P=0.6$. Conversely, $\mathrm{M} 2$ remains almost invisible up to the value $D / P=0.5$ where its contrast begins to increase; for higher $D / P$ values, the two modes become coupled, and a transfer of oscillator strength is produced from M1 toward M2. ${ }^{10}$ Eventually a situation where the two coupled plasmonic modes have similar intensity is achieved for $D / P=$ 0.65. In summary, the periodicity, $P$, controls the spectral position of the resonances, while the nanohole diameter, $D$, mainly affects the amplitude of the resonances. It is interesting to note that this behavior is in clear contrast with previous nanoribbon geometries, where the resonance frequency depends on the ribbon width, $W$, and is almost independent of the period, $P$.

The out-of-plane electric field profile of M1 and M2 modes in the far-field excitation spectra is plotted in Figure 1d. The 
(a)

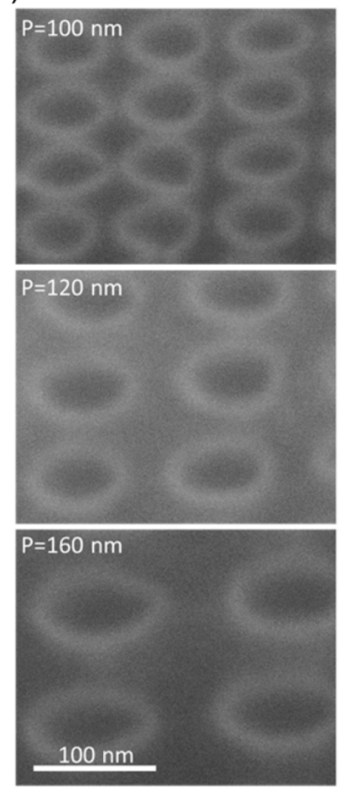

(b)
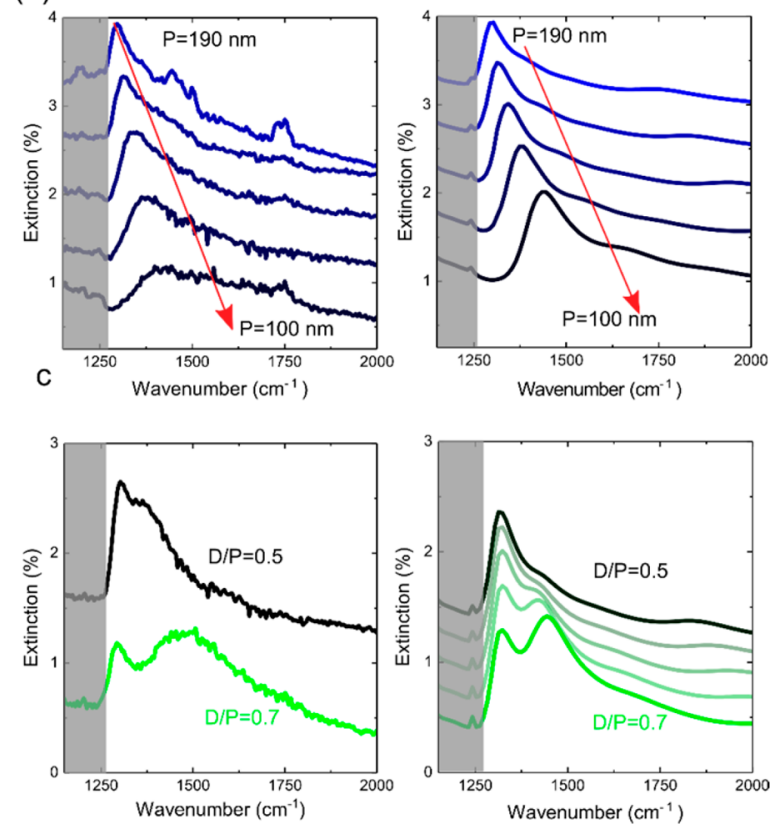

Figure 2. EBL-fabricated GNHAs. (a) SEM images of different GNHAs. (b) Experimental (left) and simulated (right) extinction spectra for GNHAs of different periods $(190,160,140,120$, and $100 \mathrm{~nm})$ and $D / P=0.5$. Experimental spectra are for unbiased graphene. For simulations, $E_{\mathrm{F}}$ $=0.25 \mathrm{eV}$ and $\tau=20 \mathrm{fs}$ are assumed. Shadowed gray areas correspond to $\mathrm{SiO}_{2}$ Reststrahlen band. Curves are vertically stacked for clarity. (c) (left) Experimental extinction spectra for GNHAs of aspect ratio $D / P=0.5$ and 0.7 and (right) simulated extinction spectra for GNHAs of aspect ratio from 0.5 to 0.7 showing the coupling of the two plasmonic modes. For simulations, $E_{\mathrm{F}}=0.3 \mathrm{eV}$ and $\tau=20$ fs is assumed. Shadowed gray areas correspond to $\mathrm{SiO}_{2}$ Reststrahlen band. Curves are vertically stacked for clarity.

field plot shows that the fundamental mode M1 is dipolar and M2 is hexapolar so that they both have a nonzero dipole moment coupling with the impinging electric field. Also, the plot of field intensity decay, $|E|^{2} /\left|E_{\max }\right|^{2}$, from the graphene surface for both M1 and M2 shows that the intensity is concentrated in about $15 \mathrm{~nm}$ from the surface, similar to what has been reported for graphene nanoribbon arrays. ${ }^{6}$ These graphene nanostructures are promising for surface-enhanced IR absorption (SEIRA) sensing as they combine easy design, extreme field confinement, and the possibility to excite multiple LSPR modes enabling multiband sensing, a feature not available in nanoribbons or other localized structures.

Graphene nanohole arrays $\left(150 \times 150 \mu \mathrm{m}^{2}\right)$ of different periods and $D / P$ ratios were fabricated on a thermally oxidized $\mathrm{SiO}_{2}$ on $\mathrm{Si}$ substrate using standard electron beam lithography (EBL) to experimentally probe the plasmonic response of the system as a function of the geometry. Figure 2 a shows the scanning electron microscope (SEM) images of different GNHA geometries.

The electromagnetic response of the system is quantified by the extinction spectrum measured by an infrared microscope coupled to a Fourier-transform infrared spectrometer (FTIR). The extinction spectrum is defined as $1-T / T_{0}$, where $T$ is the transmission through the GNHA and $T_{0}$ is the transmission spectrum of a reference area on the substrate without graphene. Figure $2 \mathrm{~b}$ shows the extinction spectra of GNHAs with different periods $(100-190 \mathrm{~nm})$ at a fixed $D / P$ ratio of 0.5 . Unlike in graphene nanoribbon structures, the coupling of light and graphene plasmons in GNHAs is polarization independent. This is advantageous for practical applications typically using unpolarized infrared sources, as it eliminates the need for a polarizing element and the 50\% reduction of the infrared signal. In Figure $2 \mathrm{~b}$, we can see that only the fundamental plasmon mode is visible and that its position blue shifts as the period is reduced, as expected from the previous discussion. Note that the lower frequency limit of the plasmonic response achievable in our experiments is $\sim 1250$ $\mathrm{cm}^{-1}(\lambda \approx 8 \mu \mathrm{m})$, which is the onset of $\mathrm{SiO}_{2}$ Reststrahlen band (represented in gray). This substrate-related limitation can be overcome using a phonon-free mid-IR substrate (e.g., $\mathrm{CaF}_{2}$ ) or changing the gate insulator (e.g., $\mathrm{Al}_{2} \mathrm{O}_{3}$ or $\left.\mathrm{SiN}\right) .{ }^{16}$ The upper limit of the plasmonic response is determined in this case by the resolution of the nanopatterning technique employed and ultimately by losses associated with interband transitions. Simulated extinction spectra for the same geometric parameters are reported in the right panel of Figure $2 b$, showing good agreement with experimental spectra in terms of tuning range and resonance contrast. A comparison of the experimental and simulated spectra for $P=100 \mathrm{~nm}$ suggests that the nanofabrication yield becomes critical for small periods affecting the quality of the plasmon response. In these simulations, $E_{\mathrm{F}}=0.25 \mathrm{eV}$ and $\tau=20 \mathrm{fs}$ for the graphene Fermi level and relaxation time, respectively, were found to best reproduce the inherent doping and mobility of the unbiased processed sample. These values are similar to those of previous graphene plasmonics experiments.

In the left panel of Figure 2c, we address experimentally the role of the $D / P$ parameter for fixed $P=190 \mathrm{~nm}$. We focus on two scenarios: for $D / P=0.5$ (black curve), only the fundamental mode $\mathrm{M} 1$ is clearly distinguished; for $D / P=$ 0.7 (green curve), both $\mathrm{M} 1$ and $\mathrm{M} 2$ are clearly visible and their intensities are comparable, as discussed in Figure 1c. The configuration where M1 and M2 are coupled is particularly relevant for applications as it allows one to have a multiband and broad plasmonic response in the mid-IR range relying on a very simple geometry. Obtaining the same with localized 
resonators (ribbons or disks) would require a complex multiresonant geometry. ${ }^{17-19}$ In the right panel of Figure $2 c$, the simulated extinction spectra are reported for the geometries considered in the experiment $(P=190 \mathrm{~nm}, D / P=0.5$ and 0.7$)$. Also, additional simulations for $D / P$ values between 0.5 and 0.7 are reported (semitransparent curves) to illustrate the transition between these two spectra and the coupling of M1 and M2 discussed previously. In these simulations, the values $E_{\mathrm{F}}=0.3 \mathrm{eV}$ and $\tau=20 \mathrm{fs}$ are used to compare with experimental data. A study of the evolution of the plasmonic line widths in GNHAs as a function of geometry is presented in the SI. The observed trend is similar to that already reported for similar graphene nanostructures on $\mathrm{SiO}_{2}$ substrate. ${ }^{20}$

One of the most appealing features of graphene for mid-IR optoelectronics is the inherent tunability of its optical conductivity via electrostatic gating. In the mid-IR range, this results in dynamically tunable plasmonic modes that can be exploited to devise reconfigurable optical metadevices. ${ }^{21}$ This fundamental feature is currently unachievable in conventional plasmonic and metamaterials based on metals and can pave the way to, for example, optical SEIRA sensors where no spectrometer is needed to achieve spectral selectivity. GNHA surfaces are extremely interesting in this sense as they can be engineered to give multiple LSPR modes in an electrically connected surface, allowing the possibility of electrostatically tuning the plasmonic response without any additional contact element (as required, for instance, in graphene nanoribbons). Figure $3 \mathrm{a}$ outlines the scheme for electrostatic tuning of the

(a)
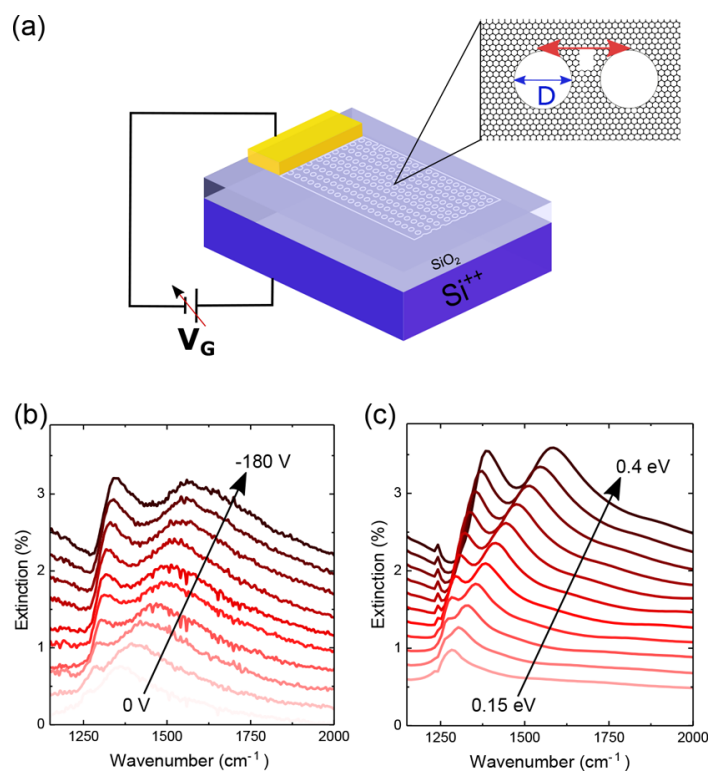

Figure 3. Electrostatic tuning of GNHAs. (a) Schematic of the electrostatic tuning of GNHA. (b) Experimental extinction spectra as a function of the gate voltage for GNHAs with $P=190 \mathrm{~nm}$ and $D / P=$ 0.7. Curves are vertically stacked for clarity. (c) Simulated extinction spectra as a function of the Fermi energy for GNHAs with $P=190$ $\mathrm{nm}$ and $D / P=0.7$. Curves are vertically stacked for clarity.

GNHA response by changing the Fermi level of graphene. Figure $3 \mathrm{~b}$ shows the experimental results for the electrostatic tuning of a GNHA surface with $P=180 \mathrm{~nm}$ and $D / P=0.65$ where two plasmonic bands of similar intensity are expected. We observe a clear spectral tuning, a progressive blue shift, and increase in intensity of the plasmonic response as the bias voltage is varied from 0 to $-180 \mathrm{~V}$. Note that at $\sim 0 \mathrm{~V}$ bias, only one of the two plasmonic modes (M2) is evident due to the proximity to the $\mathrm{SiO}_{2}$ phonon band. As the bias voltage is increased, the visibility of the fundamental mode M1 increases as it emerges from the Reststrahlen band, and for high bias voltage, the two become comparable. The simulated spectra as a function of the graphene Fermi level, $E_{F}$, for the same geometry are reported in Figure $3 \mathrm{c}$ and show a similar behavior corroborating the experimental results.

The GNHA samples of Figures 2 and 3 were fabricated using electron beam lithography (EBL), which is the preferred nanopatterning technique in academic research for test devices but constitutes an unacceptable bottleneck for large scale applications due to its low throughput and high cost. A number of mass-scalable techniques have been reported in literature for large scale fabrication of graphene nanostructures such as block copolymer method and nanosphere lithography. ${ }^{22}$ The latter has been used to fabricate large arrays of graphene antidots arranged in a hexagonal lattice. ${ }^{12}$ Although excitation of plasmon-phonon modes from such graphene antidot arrays is reported, the experimental LSPR response appears poor for real applications, and the electrostatic tunability of the plasmonic response has not been proven, which is attributed to large wafer-scale variations and poor reproducibility of these methods.

Moreover, this method allows only limited control in the shape and geometry of the nanostructures via the diameter and the self-assembled arrangement of the nanospheres. In fact, only a hexagonal lattice can be readily obtained from a close packed array of nanospheres without resorting to a prebuilt template. Instead, the free choice of the lattice symmetry is an important degree of freedom when engineering the response of a photonic or plasmonic crystal for a certain application. ${ }^{15}$ Among the other scalable nanopatterning methods, nanoimprint lithography (NIL) is the most promising for its low cost and high-throughput that satisfies industrial integration. ${ }^{23}$ Moreover, it is the best choice for large-scale periodic microand nanopatterns, such as plasmonic gratings or photonic crystals. $^{24}$

Here we propose a method to fabricate large-area graphene plasmonic nanostructures based on high throughput nanoimprint lithography (NIL) in combination with laser ablation. The detailed description of the technique is given in Methods and ref 11. In Figure 4a, we show the SEM picture of a typical GNHA fabricated by NIL. Figure $4 b, c$ depicts a comparison of the extinction spectra of two typical GNHAs fabricated with EBL and NIL under electrostatic tuning. The two surfaces have similar geometric parameters. The spectral baselines have been reported at the onset of the $\mathrm{SiO}_{2}$ Reststrahlen band for a better comparison of the plasmon tuning. The plasmonic responses measured from the two samples are similar, in terms of both peak intensities and quality factors. However, the spectra from the NIL fabricated GNHAs are affected by the vibrational fingerprints of the polymer used in the process, which can be eliminated by annealing at high temperatures.

In conclusion, we have demonstrated electrostatically tunable graphene periodic nanohole arrays working in the mid-IR. In particular, we show that nanoholes arranged in a square lattice on monolayer graphene offer the possibility of exciting multiband plasmonic resonances by properly selecting the geometric parameters. The position of these resonances can be engineered by tuning the geometrical parameters of the system. In addition, we have also demonstrated that such plasmonic crystals can be fabricated by a controllable and 
(a)

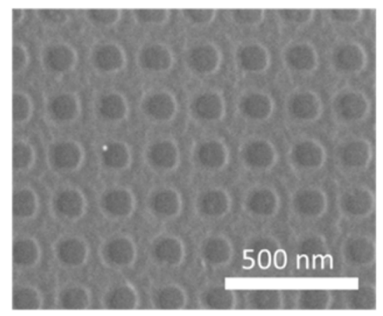

(b)

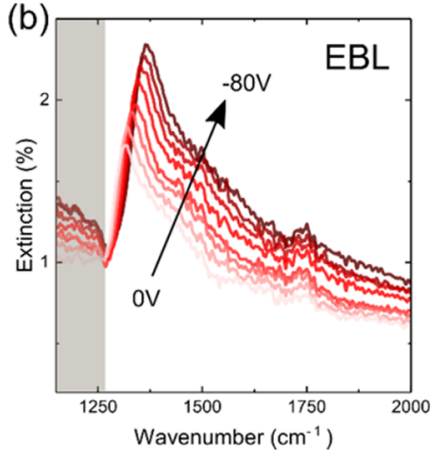

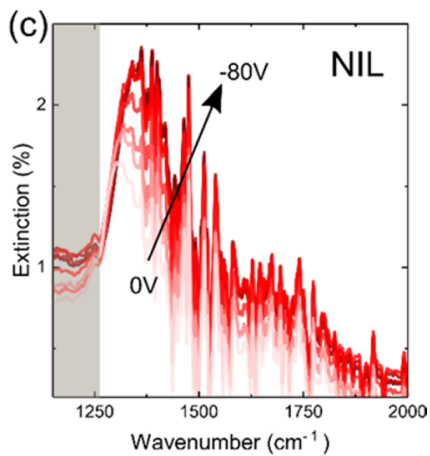

Figure 4. Comparison of EBL and NIL fabricated GNHAs. (a) SEM image of NIL fabricated GNHA. (b) Experimental extinction spectra as a function of the gate voltage for GNHAs fabricated with EBL, $P=160 \mathrm{~nm}$ and $D=80 \mathrm{~nm}$. (c) Experimental extinction spectra as a function of the

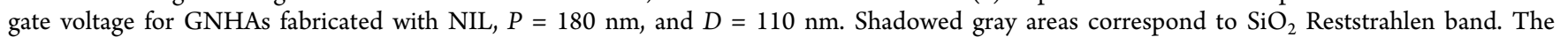
baselines of the curves have been corrected to highlight the plasmon mode tuning.

repeatable large-scale fabrication technique on wafer-scale areas.

Methods. Finite Element Simulation of GNHAs. Electromagnetic simulations in Figures 1-3 are realized using a Finite Elements Method commercial software (Ansys HFSS). A GNHA unit cell is simulated using periodic boundary conditions. Graphene is modeled as a two-dimensional surface with complex conductivity from Kubo formula. ${ }^{25} \mathrm{SiO}_{2}$ dispersion for simulated spectra in Figures 2 and 3 is taken from Kitamura. ${ }^{26}$ Transmission and reflection spectra are normalized by the response of the bare device without graphene nanostructures

Fabrication of GNHAs by EBL. Double-side polished silicon substrate with a $285 \mathrm{~nm}$ thin layer of native dry oxide $\left(\mathrm{SiO}_{2}\right)$ was used as the substrate. Graphene grown by chemical vapor deposition on a copper catalyst (from Graphenea Inc.) was wet-transferred to the $\mathrm{Si} / \mathrm{SiO}_{2}$ substrate. A $60 \mathrm{~nm}$ thick layer of electron beam resist was spin coated on the $\mathrm{Si} / \mathrm{SiO}_{2} /$ graphene chip. Subsequently, nanohole arrays with different aspect ratios were exposed using CRESTEC $50 \mathrm{keV}$ electron beam lithography system. After resist development, the nanoholes were etched by reactive ion etching using oxygen/argon plasma at $10 \mathrm{~W}$ for $60 \mathrm{~s}$. The resist was then removed using acetone, followed by isopropyl alcohol and DI water rinsing.

Fabrication of GNHAs by NIL. Graphene was grown on commercially purchased copper foil in an Aixtron Black Magic with millimeter-sized single-crystal domains. ${ }^{27}$ Prior to graphene transfer, a double side polished silicon substrate with a $285 \mathrm{~nm}$ thin layer of native dry oxide $\left(\mathrm{SiO}_{2}\right)$ had metal contacts of chrome and gold (thicknesses 5 and $45 \mathrm{~nm}$, respectively) evaporated through a shadow mask. The chip size of $20 \mathrm{~mm} \times 20 \mathrm{~mm}$ has an intended four sample areas of $5 \mathrm{~mm}$ $\times 5 \mathrm{~mm}$. A large single sheet of graphene was then transferred using standard techniques ${ }^{28}$ covering all the devices. Individual devices were then electrically separated around the perimeter of the contacts by laser ablation of unwanted graphene, ${ }^{29}$ a method that has been demonstrated not to ablate the substrate or affect the electrical properties of graphene. ${ }^{30}$ The devices were now ready for nanoimprint lithography (NIL). The chip was spin coated with $85 \mathrm{~nm}$ of $\mathrm{mr}-\mathrm{I} 7010 \mathrm{E}$ resist, baked at 125 ${ }^{\circ} \mathrm{C}$ for $60 \mathrm{~s}$, and then thermally imprinted at $6 \mathrm{bar} / 130^{\circ} \mathrm{C}$ for 10 min using a NILT technology CNIv2. The imprinted pattern used was the same electron-beam-lithography defined mask as presented in ref 11 , with four $5 \mathrm{~mm} \times 5 \mathrm{~mm}$ areas of a regular square hole pattern. The chip was then subjected to a $20 \mathrm{~W} \mathrm{O}_{2}$ plasma for $60 \mathrm{~s}$ to remove residual resist and etch the graphene. Remaining resist was removed in warm acetone, and resist residuals were removed by annealing at $225{ }^{\circ} \mathrm{C}$ for 30 min in nitrogen. ${ }^{31}$ The total processing time after graphene transfer for laser ablation, imprint lithography, and etching is less than $90 \mathrm{~min}$. More details on the characterization (terahertz time domain spectroscopy conductivity, electrical, and micro-Raman measurements) on these graphene nanohole arrays can be found in the Supporting Information.

Optical Measurements. Extinction spectra were measured with a Fourier transform IR interferometer coupled to an IR microscope (Bruker TENSOR II and Hyperion 2000). Transmission spectra were collected through a Cassegrain objective $(\mathrm{NA}=0.4,15 \times)$ and measured by a liquid-nitrogencooled mercury-cadmium-tellurium detector. Extinction spectra $\left(1-T / T_{0}\right)$ were calculated by normalizing the transmission spectrum by that of an area without graphene $\left(T_{0}\right)$. For electrostatic tuning, a bias voltage $\left(V_{\mathrm{G}}\right)$ was applied between the silicon backside and a metallic pad connected to the GNHA array.

\section{ASSOCIATED CONTENT}

\section{S Supporting Information}

The Supporting Information is available free of charge on the ACS Publications website at DOI: 10.1021/acs.nanolett.8b02613.

Plasmonic linewidth versus geometry in GNHAs and further measurements on nanoimprinted graphene nanohole arrays (PDF)

\section{AUTHOR INFORMATION}

\section{Corresponding Author}

*E-mail: valerio.pruneri@icfo.eu.

ORCID $\odot$

Kavitha K. Gopalan: 0000-0002-2570-3031

Patrick R. Whelan: 0000-0002-3978-7029

\section{Author Contributions}

The manuscript was written through contributions of all authors. All authors have given approval to the final version of the manuscript.

\section{Funding}

We acknowledge financial support from the Spanish Ministry of Economy and Competitiveness through the "Severo Ochoa" Programme for Centres of Excellence in R\&D (SEV-20150522) and OPTO-SCREEN (TEC2016-75080-R), from 
Fundació Privada Cellex, from Generalitat de Catalunya through the CERCA program, AGAUR 2017 SGR 1634 and from the European Union H2020 Programme under grant agreement (no. 696656) "Graphene Flagship". Danish National Research Foundation (DNRF) Center for Nanostructured Graphene (DNRF103), EU Horizon 2020 (3DAM, grant agreement 692527), are also acknowledged for funding.

\section{Notes}

The authors declare no competing financial interest.

\section{ACKNOWLEDGMENTS}

K.K.G. acknowledges the International $\mathrm{PhD}$ fellowship La Caixa - Severo Ochoa@ ICFO.

\section{ABBREVIATIONS}

GNHA, graphene nanohole array; EBL, electron-beam lithography; NIL, nanoimprint lithography

\section{REFERENCES}

(1) Low, T.; Avouris, P. Graphene Plasmonics for Terahertz to MidInfrared Applications. ACS Nano 2014, 8, 1086-1101.

(2) Ju, L.; Geng, B.; Horng, J.; Girit, C.; Martin, M.; Hao, Z.; Bechtel, H. A.; Liang, X.; Zettl, A.; Shen, Y. R.; et al. Graphene Plasmonics for Tunable Terahertz Metamaterials. Nat. Nanotechnol. 2011, 6 (10), 630-634.

(3) García de Abajo, F. J. Graphene Plasmonics: Challenges and Opportunities. ACS Photonics 2014, 1 (3), 135-152.

(4) Grigorenko, A. N.; Polini, M.; Novoselov, K. S. Graphene Plasmonics. Nat. Photonics 2012, 6 (11), 749-758.

(5) Yang, X.; Sun, Z.; Low, T.; Hu, H.; Guo, X.; García de Abajo, F. J.; Avouris, P.; Dai, Q. Nanomaterial-Based Plasmon-Enhanced Infrared Spectroscopy. Adv. Mater. 2018, 30 (20), 1704896.

(6) Rodrigo, D.; Limaj, O.; Janner, D.; Etezadi, D.; García De Abajo, F. J.; Pruneri, V.; Altug, H. Mid-Infrared Plasmonic Biosensing with Graphene. Science (Washington, DC, U. S.) 2015, 349 (6244), 165168.

(7) Koppens, F. H. L.; Chang, D. E.; García de Abajo, F. J. Graphene Plasmonics: A Platform for Strong Light-Matter Interactions. Nano Lett. 2011, 11 (8), 3370-3377.

(8) Yeung, K. Y. M.; Chee, J.; Yoon, H.; Song, Y.; Kong, J.; Ham, D. Far-Infrared Graphene Plasmonic Crystals for Plasmonic Band Engineering. Nano Lett. 2014, 14 (5), 2479-2484.

(9) Liu, P. Q.; Valmorra, F.; Maissen, C.; Faist, J. Electrically Tunable Graphene Anti-Dot Array Terahertz Plasmonic Crystals Exhibiting Multi-Band Resonances. Optica 2015, 2 (2), 135.

(10) Nikitin, A. Y.; Guinea, F.; Martin-Moreno, L. Resonant Plasmonic Effects in Periodic Graphene Antidot Arrays. Appl. Phys. Lett. 2012, 101 (15), 151119.

(11) Mackenzie, D. M. A.; Smistrup, K.; Whelan, P. R.; Luo, B.; Shivayogimath, A.; Nielsen, T.; Petersen, D. H.; Messina, S. A.; Bøggild, P. Batch Fabrication of Nanopatterned Graphene Devices via Nanoimprint Lithography. Appl. Phys. Lett. 2017, 111 (19), 193103.

(12) Zhu, X.; Wang, W.; Yan, W.; Larsen, M. B.; Bøggild, P.; Pedersen, T. G.; Xiao, S.; Zi, J.; Mortensen, N. A. Plasmon-Phonon Coupling in Large-Area Graphene Dot and Antidot Arrays Fabricated by Nanosphere Lithography. Nano Lett. 2014, 14 (5), 2907-2913.

(13) Liang, X.; Jung, Y. S.; Wu, S.; Ismach, A.; Olynick, D. L.; Cabrini, S.; Bokor, J. Formation of Bandgap and Subbands in Graphene Nanomeshes with Sub-10 Nm Ribbon Width Fabricated via Nanoimprint Lithography. Nano Lett. 2010, 10 (7), 2454-2460.

(14) Jin, D.; Christensen, T.; Soljačić, M.; Fang, N. X.; Lu, L.; Zhang, X. Infrared Topological Plasmons in Graphene. Phys. Rev. Lett. 2017, 118 (24), 245301.
(15) Yeung, K. Y. M.; Chee, J.; Song, Y.; Kong, J.; Ham, D. Symmetry Engineering of Graphene Plasmonic Crystals. Nano Lett. 2015, 15 (8), 5001-5009.

(16) Jang, M. S.; Brar, V. W.; Sherrott, M. C.; Lopez, J. J.; Kim, L.; Kim, S.; Choi, M.; Atwater, H. A. Tunable Large Resonant Absorption in a Midinfrared Graphene Salisbury Screen. Phys. Rev. B: Condens. Matter Mater. Phys. 2014, 90 (16), 165409.

(17) Farmer, D. B.; Rodrigo, D.; Low, T.; Avouris, P. PlasmonPlasmon Hybridization and Bandwidth Enhancement in Nanostructured Graphene. Nano Lett. 2015, 15 (4), 2582-2587.

(18) Rodrigo, D.; Low, T.; Farmer, D. B.; Altug, H.; Avouris, P. Plasmon Coupling in Extended Structures: Graphene Superlattice Nanoribbon Arrays. Phys. Rev. B: Condens. Matter Mater. Phys. 2016, 93 (12), 125407.

(19) Low, T.; Avouris, P. Graphene Plasmonics for Terahertz to Mid-Infrared Applications. ACS Nano 2014, 8 (2), 1086.

(20) Yan, H.; Low, T.; Zhu, W.; Wu, Y.; Freitag, M.; Li, X.; Guinea, F.; Avouris, P.; Xia, F. Damping Pathways of Mid-Infrared Plasmons in Graphene Nanostructures. Nat. Photonics 2013, 7 (5), 394-399.

(21) Zheludev, N. I.; Kivshar, Y. S. From Metamaterials to Metadevices. Nat. Mater. 2012, 11 (11), 917-924.

(22) Cagliani, A.; Mackenzie, D. M. A.; Tschammer, L. K.; Pizzocchero, F.; Almdal, K.; Bøggild, P. Large-Area Nanopatterned Graphene for Ultrasensitive Gas Sensing. Nano Res. 2014, 7 (5), $743-$ 754.

(23) Kooy, N.; Mohamed, K.; Pin, L. T.; Guan, O. S. A Review of Roll-to-Roll Nanoimprint Lithography. Nanoscale Res. Lett. 2014, 9 (1), 320 .

(24) Kurosawa, H.; Choi, B.; Sugimoto, Y.; Iwanaga, M. HighPerformance Metasurface Polarizers with Extinction Ratios Exceeding 12000. Opt. Express 2017, 25 (4), 4446.

(25) Falkovsky, L. A. Optical Properties of Graphene. J. Phys. Conf. Ser. 2008, 129, 012004.

(26) Kitamura, R.; Pilon, L.; Jonasz, M. Optical Constants of Silica Glass from Extreme Ultraviolet to Far Infrared at near Room Temperature. Appl. Opt. 2007, 46 (33), 8118.

(27) Luo, B.; Caridad, J. M.; Whelan, P. R.; Thomsen, J. D.; Mackenzie, D. M. A.; Grubišić Čabo, A.; Mahatha, S. K.; Bianchi, M.; Hofmann, P.; Jepsen, P. U.; et al. Sputtering an Exterior Metal Coating on Copper Enclosure for Large-Scale Growth of SingleCrystalline Graphene. 2D Mater. 2017, 4 (4), 045017.

(28) Whelan, P. R.; Jessen, B. S.; Wang, R.; Luo, B.; Stoot, A. C.; Mackenzie, D. M. A.; Braeuninger-Weimer, P.; Jouvray, A.; Prager, L.; Camilli, L.; et al. Raman Spectral Indicators of Catalyst Decoupling for Transfer of CVD Grown 2D Materials. Carbon 2017, 117, 75-81.

(29) Mackenzie, D. M. A.; Buron, J. D.; Whelan, P. R.; Jessen, B. S.; Silajdźić, A.; Pesquera, A.; Centeno, A.; Zurutuza, A.; Bøggild, P.; Petersen, D. H. Fabrication of CVD Graphene-Based Devices via Laser Ablation for Wafer-Scale Characterization. 2D Mater. 2015, 2 (4), 045003.

(30) Mackenzie, D. M. A.; Buron, J. D.; Bøggild, P.; Jepsen, P. U.; Petersen, D. H. Contactless Graphene Conductance Measurements: The Effect of Device Fabrication on Terahertz Time-Domain Spectroscopy. Int. J. Nanotechnol. 2016, 13 (8/9), 591.

(31) Gammelgaard, L.; Caridad, J. M.; Cagliani, A.; MacKenzie, D. M. A.; Petersen, D. H.; Booth, T. J.; Bøggild, P. Graphene Transport Properties upon Exposure to PMMA Processing and Heat Treatments. 2D Mater. 2014, 1 (3), 035005. 